\title{
An investigation of dynamic mechanical behaviour of Ti6Al4V titanium alloy at room temperature
}

\author{
Chun Ran, Pengwan Chen, Baoqiao Guo, Ling Li, and Haibo Liu \\ State Key Laboratory of Explosion Science and Technology, Beijing Institute of Technology, Beijing100081, China
}

\begin{abstract}
To study the high strain rate shear behaviour of Ti6Al4V titanium alloy, a series of dynamic compression experiments has been performed by split Hopkinson pressure bar (SHPB) using Flat Hat-shaped specimen at room temperature. Macro true shear stress-true strain curves were obtained under different strain rate loading conditions at room temperature. The effects of strain hardening and strain rate hardening on the dynamic mechanical properties of Ti6Al4V titanium alloy were discussed. Results indicate that a) The higher the strain rate, the higher the flow stress, therefore, the material has obvious strain rate hardening effect, b) It is ductile failure for Ti6Al4V titanium alloy under quasi-static loading condition, c) For dynamical tests, the values for true shear stress increase with increasing true strain till the maximum true shear stress, on the contrary, the values for true shear stress decrease with increasing the true strain after the maximum true shear stress and d) The flow stress increases with increasing the true strain under quasi-static loading condition during the plastic deformation.
\end{abstract}

\section{Introduction}

Ti-6Al $-4 \mathrm{~V}$, an $\alpha+\beta$ titanium alloy, is world widely used to manufacture the engine fan and blade in aerospace industries due to its highly attractive material parameters, such as low mass density, high strength and high temperature stability ([1]). A profusion of studies on the dynamic mechanical behaviour of titanium and titanium alloys have been carried out ([2]). Chichili et al ([3]) and Dong et al ([4], [5]) investigated dynamic mechanical behaviour of $\alpha-\mathrm{Ti}$ at room temperature by using split Hopkinson pressure bar (SHPB) and found that the strain hardening is strain rate and temperature dependence. Rittel et al ([6]-[9]) proposed a new failure criterion based on mechanical energy (the stored energy of cold work as the driving force for microstructural rearrangement by DRX) rather than a critical strain proposed by Recht ([10]). A series of corresponding work have been carried out by Bai, Meyer, Xue, Peirs, Staker ([11]-[15]). Hat-shaped (HS) specimen was invented in 1977 by Hartmann and Meyer at the Fraunhofer Institute (IFAM, Bremen, Germany). Due to the unique geometry of HS specimen, extremely high strains at high strain-rate can be reached. However, in fact, the shear stress measurement is influenced by edge effect, and it is impossible to track the shear band, detect its temperature and measure the local strain. To reduce edge effect and measure the temperature rise during the deformation, the Flat Hat-shaped (FHS) specimen was designed by Clos ([16, [17]).

In this paper, the dynamic mechanical behaviour of Ti6A14V titanium alloy at room temperature has been studied by FHS specimen. The purpose of this work is to bring recent experimental results on the dynamic mechanical behaviour of Ti6Al4V titanium alloy. The paper is organized as follows: First, the experimental techniques are introduced. Next, experimental results and discussion are presented, followed by concluding remarks.

\section{Experimental techniques}

The Ti6Al4V titanium alloy is provided by Beijing Institute of Aerial Materials. The split Hopkinson pressure bar technique is used to load the specimens dynamically. The FHS specimen is sandwiched between two bars (Fig. 1). An impact pulse is provided with a striker driven by a light gas gun. It should be noted that the setup used in this work is nickel $(18 \mathrm{Ni})$ bars with diameters of $14 \mathrm{~mm}$ and the lengths of the striker, incident and transmission bar are $0.2 \mathrm{~m}, 1.2 \mathrm{~m}, 1.2 \mathrm{~m}$, respectively. The dynamic mechanical behaviour of Ti6Al4V titanium alloy at room temperature under $4000 \sim 7400 \mathrm{~s}^{-1}$ strain rate loading conditions have been measured. Meanwhile, the quasi-static properties of Ti6Al4V at room temperature have been performed using MTS test machine (INSTRON 5985) based on GB/T 7314-2005. It should be noted that the strain rate is $10^{-3} \mathrm{~s}^{-1}$ and at least three samples were tested for each strain rate loading condition, being always found good reproducibility of results. Also it should be pointed out that the cylindrical specimens, $6 \mathrm{~mm}$ in diameter and $10 \mathrm{~mm}$ in length, were used in the quasi-static tests.

Fig. 2 shows the in-plane dimensions of the FHS specimen used in this study. In an axi-symmetric FHS 
specimen, shear strains are concentrated in a narrow region (shown in Fig. 3). The specimen can be divided into three parts: the upper hat part, the lower edge part and the shear region where extremely high strain rate, strain and temperature generate and propagate.

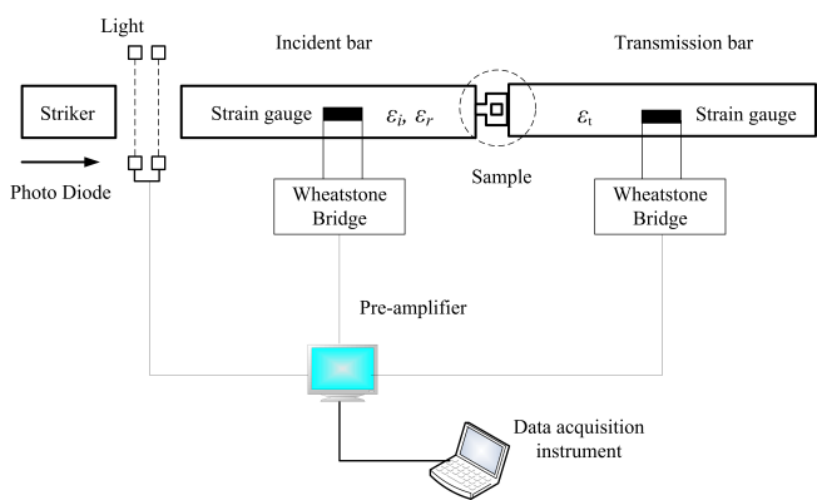

Figure 1. Sketch of a split Hopkinson pressure bar set-up
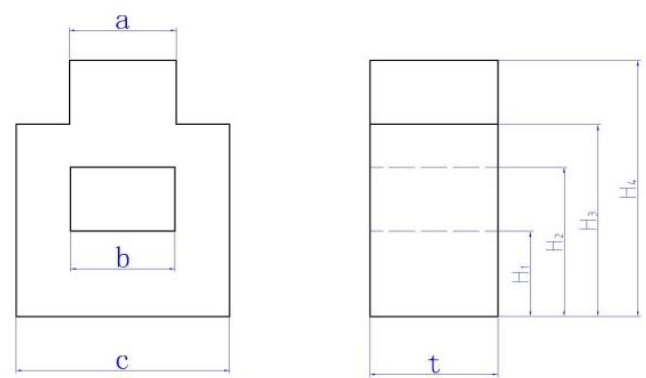

Figure 2. In-plane dimensions of FHS specimen : $a=6 \mathrm{~mm}$, $\mathrm{b}=5.6 \mathrm{~mm}, \mathrm{c}=13 \mathrm{~mm}, \mathrm{H} 1=3 \mathrm{~mm}, \mathrm{H} 2=6 \mathrm{~mm}, \mathrm{H} 3=9 \mathrm{~mm}, \mathrm{H} 4=11$ $\mathrm{mm}, \mathrm{t}=6 \mathrm{~mm}$.

When one-dimensional stress-waves in the bars are achieved and the specimen is in a state of uniform stress, the strain rate, strain and stress histories in the specimen can be computed as:

$$
\begin{gathered}
\dot{\varepsilon}_{s}(t)=-2 \frac{C_{0}}{h} \varepsilon_{r}(t) \\
\varepsilon_{s}(t)=-2 \frac{C_{0}}{h} \int_{0}^{t} \varepsilon_{r}(t) d t \\
\sigma_{s}(t)=\frac{A_{0}}{A_{s}} E_{0} \varepsilon_{r}(t)
\end{gathered}
$$

where $A_{0}$ is the cross-sectional of the bars, $E_{0}$ and $C_{0}$ are Young's modulus and elastic bar wave speed in the bar material, respectively. Here, $A s$ and $h$ are the initial crosssectional area and thickness of deformation section of the FHs specimen, respectively. And $\varepsilon_{r}(t)$ and $\varepsilon_{t}(t)$ represent reflected and transmitted strain histories in the bars at the specimen ends, respectively.

The stress and strain in the compression-shear section of the FHS specimens can be estimated approximately, although they are complicated. As shown in Fig. 3, the load $F$ can be considered as two parts, compression and shear. Then the loading, shear stress, displacement and strain histories in the specimen can be computed as follows:

$$
\begin{gathered}
F_{s}(t)=\frac{E_{0} A_{0} \varepsilon_{t}(t)}{2} \\
\tau_{s}(t)=\frac{E_{0} A_{0} \varepsilon_{t}(t)}{2 A_{s}} \\
A_{s}=\sqrt{(a-b)^{2}+\left(H_{3}-H_{2}\right)^{2}} \times t \approx h t \\
\Delta l_{s}(t)=-2 C_{0} \int_{0}^{t} \varepsilon_{r}(t) d t \\
\gamma_{s}(t) \approx \Delta l / h=-2 C_{0} \int_{0}^{t} \varepsilon_{r}(t) d t / h
\end{gathered}
$$

Here $t$ is the thickness of the FHS specimen.

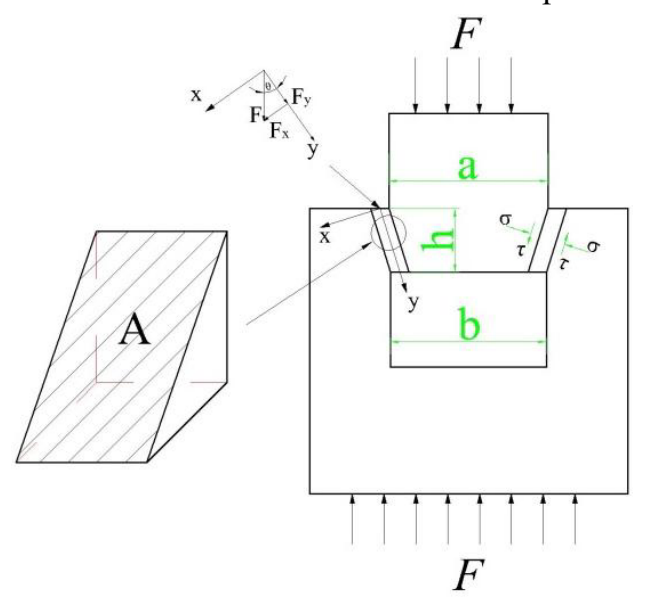

Figure 3. Schematic plot of stress state of the FHS specimen: $\mathrm{h}=\mathrm{H} 3-\mathrm{H} 2=3 \mathrm{~mm}, \mathrm{a}=6 \mathrm{~mm}, \mathrm{~b}=5.6 \mathrm{~mm}$. A denotes the area of the shear region

\section{Results and discussion}

The typical stress wave tested by gauges on split Hopkinson pressure bars at room temperature is shown in Fig. 4. Then the true shear stress-true strain can be calculated based on eqs.(2)-(6).

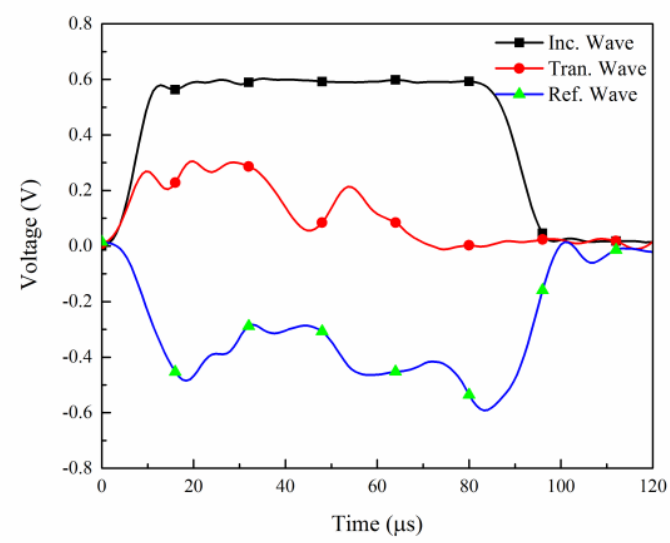

Figure 4. Stress wave tested by gauges on Hopkinson bars

\subsection{Stress and strain curves}


The resultant true shear stress - true strain for 6 different strain rate, $\varepsilon=0.001,4100,4800,5400,6600$ and $7400 \mathrm{~s}^{-1}$ are plotted in Fig. 5. It is seen that, the yield stress is about $350 \mathrm{MPa}$ for quasi-static loading $\left(\varepsilon=0.001 \mathrm{~s}^{-1}\right)$ condition and it is about $550 \mathrm{MPa}$ for $7400 \mathrm{~s}^{-1}$ loading condition. The yield stress increases apparently with increasing of the strain rate. Therefore, the strain rate hardening effect for Ti6Al4V titanium alloy is very obviously.

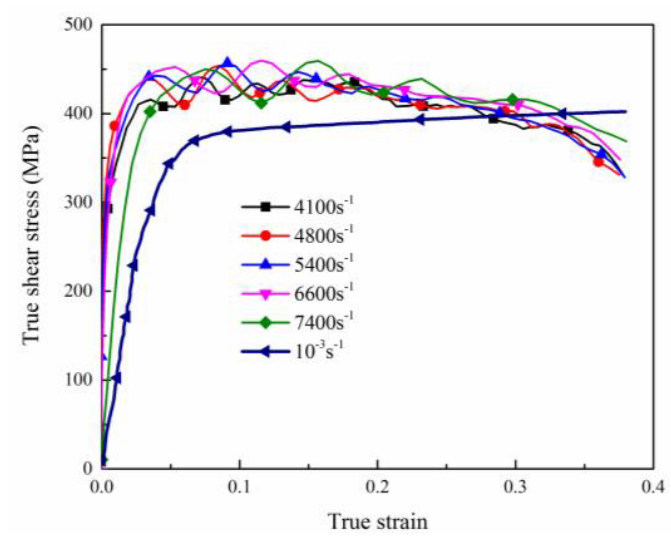

Figure 5. The true shear stress-true strain curves under different strain rate loading conditions at room temperature.

The typical true shear stress and strain rate as functions of true strain for Ti6Al4V titanium alloy at room temperature is shown in Fig.6. It is seen that, a) the maximum true shear stress is located at true strain $\gamma=0.12$. Then, the true shear stress decreases with increasing the true strain, b) the value for strain rate becomes approximately constant when the true strain $\gamma \geq 0.12$ and c) the shear failure occurs when the true strain $\gamma=0.36$.

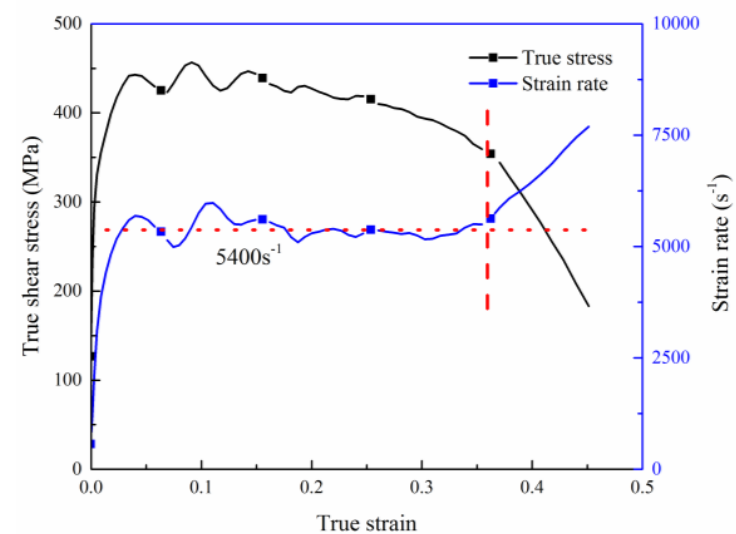

Figure 6. Typical true shear stress and strain rate as functions of true strain for Ti6Al4V titanium alloy at room temperature, the strain rate is $5300 \mathrm{~s}^{-1}$.

As can be seen from Figs. 5 and 6, for dynamical tests $\left(\varepsilon=4000 \sim 7400 \mathrm{~s}^{-1}\right)$, the values for true shear stress increase with increasing true strain till the maximum true shear stress, on the contrary, the values for true shear stress decrease with increasing the true strain after the maximum true shear stress. That is, when the strain rate is fixed, such as $\varepsilon=5300 \mathrm{~s}^{-1}$, a) for the former part, the strain hardening effect is the much higher than the thermal soften effect so that the flow stress increases with increasing the true strain and b) for the latter part, the thermal soften effect is much higher than the strain hardening effect so that the flow stress decreases with increasing the true strain. It should be pointed out that the flow stress increases with increasing the true strain when strain rate $\varepsilon=0.001 \mathrm{~s}^{-1}$ during the whole plastic deformation.

\subsection{Fractography}

Fig.7 is the typical macro-morphology of Ti6Al4V titanium alloy after dynamic compression, and the typical micro-morphology of Ti6Al4V titanium alloy after quasistatic compression is shown in Fig.8. Fig.8 shows the ductile dimples areas, it should be pointed out that there are some elongated dimples along the maximum shear stress direction. Therefore, it is ductile failure for Ti6Al4V titanium alloy under quasi-static loading condition.

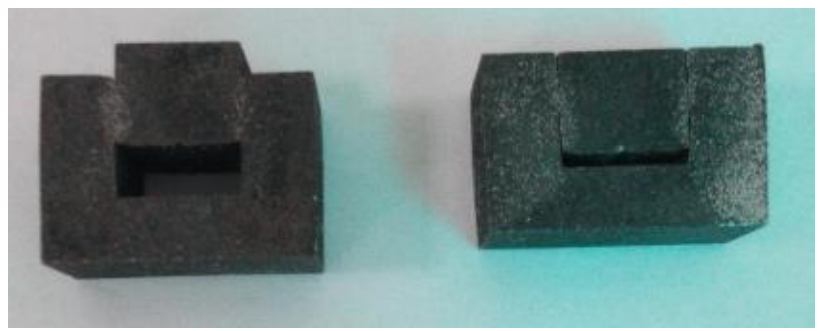

Figure 7. The typical macro-morphology of Ti6A14V titanium alloy after dynamic compression. The strain rate is $5300 \mathrm{~s}^{-1}$.

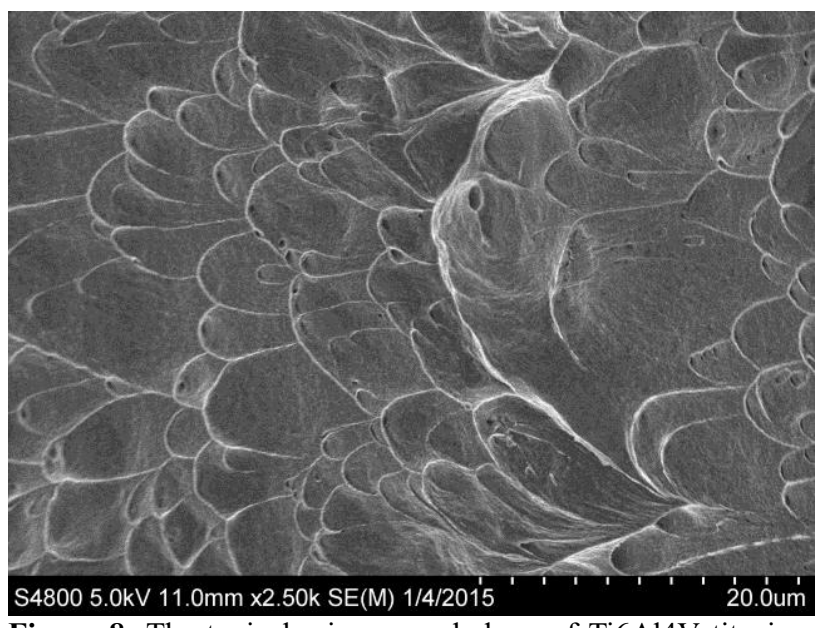

Figure 8. The typical micro-morphology of Ti6Al4V titanium alloy after quasi-static compression. The strain rate is $0.001 \mathrm{~s}^{-1}$.

\section{Conclusions}

To study the high strain rate shear behaviour of Ti6Al4V titanium alloy, a series of dynamic compression experiments has been performed by split Hopkinson pressure bar (SHPB) using Flat Hat-shaped specimen at room temperature. And the quasi-static compression tests have been performed by MTS test machine (INSTRON 5985) based on GB/T 7314-2005. The following conclusions can be drawn from the present work:

1) The higher the strain rate, the higher flow stress of Ti6Al4V titanium alloy, therefore, the material has obvious strain rate hardening effect. 
2) For dynamical tests, the values for true shear stress increase with increasing true strain till the maximum true shear stress, then, on the contrary, the values for true shear stress decrease with increasing the true strain after the maximum true shear stress.

3) The flow stress increases with increasing the true strain when strain rate $\varepsilon=0.001 \mathrm{~s}^{-1}$ during the whole plastic deformation.

4) It is ductile failure for Ti6Al4V titanium alloy under quasi-static loading condition.

\section{References}

1. M.K. McQuilan, Metall. Trans. A. 7A, 22 (1944)

2. Y. B. Xu, Y.L. Bai, Y. J. Chen. Mater Sci Eng A, 299(1), 287 (2001).

3. D.R. Chichili, K.T.Ramesh, K.J. Hemker. Acta Mater., 46(3), 189 (1998)

4. X.L. Dong, Y.Q. Fu. Acta Armamentarii. 35(7), 1016 (2014).

5. Y.Q. Fu, X.L. Dong. Rare Metal Materials and Engineering, 45(1), 102 (2016).
6. D. Rittel, Z.G. Wang, M. Merzer. Physical review letters, 96(7), 075502 (2006).

7. D Rittel. Journal of Physics D: Applied Physics, 42(21), 214009 (2009).

8. D. Rittel, P. Landau, A. Venkert. Physical review letters, 101(16), 165501 (2008).

9. D. Rittel, Z.G. Wang. Mech. Mater. 40(8), 629 (2008)

10. R. Recht. Journal of Applied Mechanics, 31(2), 189 (1964)

11. L.H. Dai, Y.L. Bai. Int. J. Impact Eng., 35(8), 704 (2008).

12. F. Pursche, L.W. Meyer. Engineering transactions, 59(2), 67 (2011)

13. Q. Xue, M.A. Meyers, V.F. Nesterenko. Mater Sci Eng A, 384, 35 (2004).

14. J. Peirs, P. Verleysen, J. Degrieck, et al. Int. J. Impact Eng., 37(6), 703 (2010).

15. M.R. Staker. Acta Metallurgica, 29(4), 683 (1981)

16. R. Clos, U. Schreppel, P. Veit, J. Phys. IV France, 10, 257 (2000).

17. R. Clos, U. Schreppel, P. Veit, J. Phys. IV France, 110,111 (2003). 